\title{
Novel association between asthma and osteoarthritis: a nationwide health and nutrition examination survey
}

Hyeon-Kyoung Koo ${ }^{1+}$, Pamela Song ${ }^{2+}$ and Joo-Hyun Lee ${ }^{3^{*}}$ (1)

\begin{abstract}
Background: Asthma and osteoarthritis $(\mathrm{OA})$ are medical conditions that inhibit physical activity and adversely affect quality of life. Despite the high prevalence, there are limited studies focusing on the comorbid condition and association between asthma and OA. The aim of this study was to assess the prevalence of OA co-occurring with asthma and to identify the relevant clinical considerations.

Methods: Adult participants aged over 40 years who completed questionnaire assessments and spirometry tests were enrolled from the Korean National Health and Nutrition Examination Survey. Asthma and OA were defined based on the medical history of a diagnosis made by a doctor. Radiographic severities of OA were measured using the Kellgren-Lawrence grading system. Chronic obstructive pulmonary disease (COPD), as a comparative respiratory disease, was diagnosed based on the spirometric results.

Results: A total of 9344 subjects were enrolled, and the prevalence of asthma and COPD were $4.6 \% \pm 0.3 \%$ and $12.0 \% \pm 0.5 \%$, respectively. The prevalence of $\mathrm{OA}$ in the asthma group was $31.9 \% \pm 2.8 \%$, which was significantly higher than that in the COPD $(17.8 \% \pm 1.5 \%)$ or control $(16.2 \% \pm 0.6 \%)$ groups. OA was more prevalent in patients with asthma after adjusting for age, sex, body mass index, and smoking status (OR 1.65; 95\% Cl 1.27-2.13). Furthermore, after adjustment of this model for the prescription of OA medication, OA remained independently associated with asthma (OR 1.56; 95\% Cl 1.10-2.20). Conversely, the relationship of OA medication with asthma was not significant $(P=0.64)$. This relationship was evident in patients with asthma without airflow limitation measured by spirometry (OR 1.97; 95\% Cl 1.32-2.93). Moreover, the radiographic severity of knee OA correlated with asthma (OR 1.10; 95\% Cl 1.0-1.21).
\end{abstract}

Conclusions: OA shows a high prevalence in patients with asthma, higher than in patients with COPD or the controls. The comorbid characteristics of these two conditions need to be considered in clinical practice.

Keywords: Asthma, Osteoarthritis, COPD, KNHANES

*Correspondence: jh19325@gmail.com

${ }^{\dagger}$ Hyeon-Kyoung Koo and Pamela Song authors contributed equally to this work

${ }^{3}$ Division of Rheumatology, Department of Internal Medicine, Inje University Ilsan Paik Hospital, Goyang 10380, Korea

Full list of author information is available at the end of the article

\section{Background}

The World Health Organization estimates that approximately 300 million people globally have asthma [1-3]. The Global Initiative for Asthma reports that the prevalence of this disease ranges from 1 to $18 \%$ in different countries [3], and it accounts for about $1 \%$ of all disability-adjusted life years lost [4]. Asthma is characterized by chronic airway inflammation with variable airflow 
limitation, and factors such as genetics and environmental variations may contribute to its development [3]. Recent reports have suggested that patients with severe asthma who require systemic glucocorticoid therapy have a higher rate of comorbidities, including osteoporosis, functional dyspepsia, cataracts, and obstructive sleep apnea [5]. However, apart from atopic disease, the risk factors for and comorbidities related to asthma are largely unknown.

Osteoarthritis (OA) is a degenerative joint disease that causes structural joint damage and pain. In US adults aged 25 years or older, the prevalence of OA of the hand, hip, or knee joint increased from 21 million in 1995 to 27 million in just over a decade [6]. OA is prevalent in female patients, its prevalence increases with age ( $\geq 50$ years), and it more frequently affects the joints of the hands and knees [6]. Older patients with joint pain have limited physical activity that indeed affects their quality of life (QOL) [7].

Owing to the high prevalence of asthma and OA, they are commonly encountered as comorbid conditions in clinical practice. However, studies aimed at identifying the association between asthma and OA are limited. The underlying etiologies of these two conditions differ, although they have synergistic effects in hindering physical activity. In OA and asthma, physical activity is limited due to joint pain and dyspnea, respectively; thus, both conditions lead to a deterioration in the QOL. However, this comorbidity may be under-recognized in those who prefer a sedentary lifestyle to cope with joint pain or dyspnea. Furthermore, there is concern that non-steroidal anti-inflammatory drugs (NSAIDs), which are commonly prescribed to patients with OA, may aggravate the symptoms of asthma [8]. Therefore, examining the prevalence and relationship of $\mathrm{OA}$ and asthma is essential to understand their clinical implications on aspects such as physical activity and QOL. This study aimed to determine the prevalence of OA co-occurring with asthma and to identify the characteristics of these comorbid conditions. The presence of OA was determined in healthy adults, patients with asthma, and patients with chronic obstructive pulmonary disease (COPD) - COPD was selected as a comparative respiratory disease of asthma. Data from the Korean National Health and Nutrition Examination Survey (KNHANES) were used for analyses.

\section{Methods}

\section{Study design and participants}

The KNHANES is a collection of nationally representative, cross-sectional, population-based health and nutritional surveys conducted by the Korean Centers for Disease Control and Prevention (KCDC) since 1998. The participants were selected using proportional allocation system sampling with multiple stratifications based on geographical area, age, and sex. The KNHANES includes a health interview, physical examination, laboratory tests, and nutritional questionnaire. All subjects who completed the questionnaire, laboratory tests, and a pulmonary function test were selected. The entire survey population participated voluntarily and provided written informed consent. The KNHANES protocol was approved by the institutional review board of the KCDC. The date is available under following web address (https ://knhanes.cdc.go.kr/knhanes/sub03/sub03_02_05.do).

\section{Definition}

The KNHANES includes a self-reported questionnaire including the following items for various diseases: "Have you been diagnosed with the disease by a doctor?" (Yes/ No) and "Do you take medicine or treatment for the disease?" (Yes/No) - asthma and OA were defined based on the responses regarding the individual's history of a medical diagnosis. Patients with asthma were divided into two groups according to the presence of airflow limitation on spirometry (forced expiratory volume in $1 \mathrm{~s}\left[\mathrm{FEV}_{1}: \mathrm{L}\right] /$ forced vital capacity $\left.[\mathrm{FVC}: \mathrm{L}]<0.7\right)$. COPD was defined when spirometry revealed airway obstruction $\left(\mathrm{FEV}_{1} / \mathrm{FVC}<0.7\right)$ among adults $\geq 40$ years of age without a history of asthma according to the Global Initiative for Chronic Obstructive Lung Disease (GOLD) guidelines [9]. Other comorbidities, including hypertension, diabetes, hypercholesterolemia, and obesity, were defined based on the KNHANES protocol. Hypertension was defined as a systolic blood pressure $\geq 140 \mathrm{mmHg}$, diastolic blood pressure $\geq 90 \mathrm{mmHg}$, or if the patient was using anti-hypertensive medication [10]. Diabetes was defined as a fasting blood glucose level of $\geq 126 \mathrm{mg} /$ $\mathrm{dL}$ or a hemoglobin A1c of $\geq 6.5 \%$, or if the patient was using treatment for diabetes [11]. Hypercholesterolemia was defined as a total cholesterol level of $>240 \mathrm{mg} / \mathrm{dL}$ or if the patient was using lipid-lowering agents [12], while obesity was defined as a body mass index (BMI) greater than $25 \mathrm{~kg} / \mathrm{m}^{2}$ based on the World Health Organization's recommendations for the Asian population [13].

\section{Measurements}

Spirometry tests were conducted in subjects aged $\geq 40$ years by using standardized equipment (model 1022; SensorMedics Corp, BD, Franklin Lakes, NJ, USA) according to the guidelines of the American Thoracic Society/European Respiratory Society [14]. Spirometry tests were repeated at least three times to ensure reproducibility and validity. The calculation for predicted values was based on the predictive equation for the Korean population [15]. To evaluate OA, radiography of the joint was performed in participants $>50$ years of 
age using a SD3000 Synchro Stand (Accele Ray, Switzerland). Anteroposterior and lateral plain radiographs of the knee, hip, and spine were acquired. Radiographic changes related to OA in each joint were independently assessed by two trained radiologists using the KellgrenLawrence (KL) grading system [16, 17]. If, for the same case, the KL grades provided by the two radiologists differed, the higher grade was accepted. If the difference comprised more than one grade, a third radiologist was consulted and the grade concordant with the third grade was accepted. A radiographic knee $K L$ grade $\geq 2$ in one or both knees was defined as radiographic knee OA. In addition, all patients described their current symptoms related to the sites of pain. QOL was measured using the validated Korean version of the self-administered EuroQOL Five Dimensions Questionnaire (EQ-5D) [18].

\section{Statistical analysis}

The KNHANES was designed using a complex, stratified, multistage probability-sampling model, and data were analyzed via the complex-sample design to represent the prevalence in the Korean national population using SAS version 9.3 and $R$ version 3.6.0. Data were presented as mean \pm standard error, or frequency (\%). In order to compare the characteristics of each subgroup, generalized linear regression was used for continuous variables and logistic regression was used for categorical variables. Models testing for an association between OA and diseases, including asthma and COPD, were adjusted for age, sex, BMI, and current smoking status (model 1), and were further adjusted based on whether OA medication was prescribed (model 2). Models testing for an association between the pain site or severity of OA, based on the radiographic KL grade, and each relevant disease were adjusted for age, sex, BMI, and current smoking status. A $P$-value $<0.05$ was used to indicate statistical significance.

\section{Results}

\section{Characteristics of the participants}

The completed questionnaire and spirometry data for a total of 9344 subjects aged $\geq 40$ years from 2010 to 2012 were retrieved from the KNHANES. Among these, 425 patients had self-reported asthma (prevalence, $4.6 \% \pm 0.3 \%$ ), and 1131 had COPD based on the spirometric measurements (prevalence, $12.0 \% \pm 0.5 \%$ ). In the asthma group, 161 patients (prevalence, $1.7 \% \pm 0.2 \%$ ) showed airflow limitation on spirometry (Fig. 1 and Additional file 1: Figure S1). The demographic and clinical characteristics of patients with either one of the two respiratory diseases-asthma or COPD—were compared to those of the control group (Table 1). The age of the patients in the asthma group was higher than that in the control group, but lower than that in the COPD group.

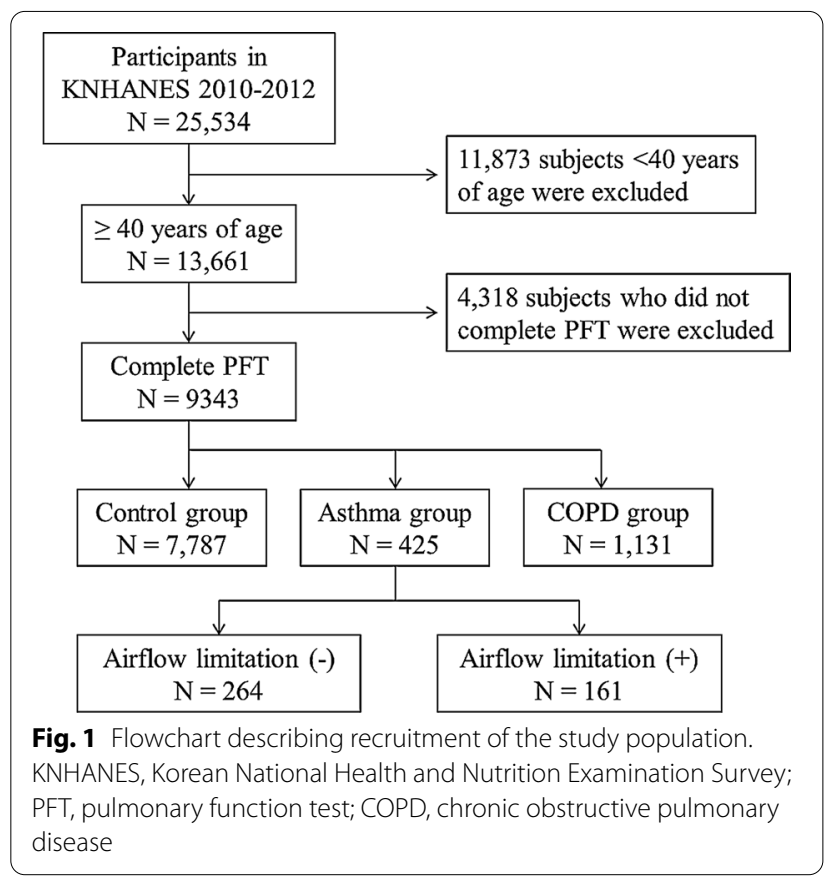

In comparison to patients with COPD, the asthma group included more females and more patients with obesity. Patients with asthma had comorbid hypertension more frequently than the control group, but less frequently than the COPD group. However, the prevalence of other metabolic syndromes, such as diabetes or hypercholesterolemia, did not significantly differ from that in the control group. Patients with asthma presented with the lowest FVC, and those with COPD had the lowest FEV In addition, QOL measured by the EQ-5D was the lowest in the asthma group (Table 1).

\section{Association between asthma and osteoarthritis}

Among adults aged $\geq 40$ years, 1190 patients (prevalence, $17.0 \% \pm 0.5 \%$ ) were diagnosed with OA by a doctor. The prevalence of OA was higher in patients with asthma $(31.9 \% \pm 2.8 \%)$ than in those with COPD $(17.8 \% \pm 1.5 \%)$ or the control group (16.2\% $\pm 0.6 \%$; Fig. 2$)$. In the univariate analysis, OA was significantly associated with asthma (OR, 2.15; 95\% CI, 1.70-2.72), but not with COPD $(P=0.34)$. The association between asthma and OA was significant regardless of the presence of airflow limitation on spirometry, although the effects were attenuated in patients with an airflow limitation (OR for asthma without airflow limitation, 2.36; 95\% CI, 1.77-3.14; OR for asthma with airflow limitation, 1.72; 95\% CI, 1.16-2.54). After adjusting for confounding factors such as age, sex, BMI, and smoking status (model 1), OA remained significantly associated with asthma (OR, 1.65; 95\% CI, $1.27-2.13)$, but not with COPD $(P=0.40)$. To identify 
Table 1 Baseline characteristics comparing patients with asthma and COPD with control groups

\begin{tabular}{|c|c|c|c|c|c|c|}
\hline & \multirow{2}{*}{$\begin{array}{l}\text { Controls } \\
(\mathrm{N}=7787)\end{array}$} & \multirow{2}{*}{$\begin{array}{l}\text { Asthma } \\
(\mathrm{N}=425)\end{array}$} & \multirow{2}{*}{$\begin{array}{l}\text { COPD } \\
(N=1131)\end{array}$} & \multicolumn{3}{|l|}{ P-value } \\
\hline & & & & Control vs. Asthma & Control vs. COPD & Asthma vs. COPD \\
\hline Prevalence, \% & $83.4 \pm 0.6$ & $4.6 \pm 0.3$ & $12.0 \pm 0.5$ & & & \\
\hline Age, mean $\pm S D$ & $54.2 \pm 0.2$ & $60.6 \pm 0.9^{*}$ & $64.7 \pm 0.4^{*}$ & $<0.001$ & $<0.001$ & $<0.001$ \\
\hline Male sex, $\%$ & $44.0 \pm 0.6$ & $43.8 \pm 2.9$ & $76.3 \pm 1.7^{*}$ & 0.93 & $<0.001$ & $<0.001$ \\
\hline $\mathrm{BMI}, \mathrm{kg} / \mathrm{m}^{2}$ & $24.4 \pm 0.04$ & $24.4 \pm 0.2$ & $23.6 \pm 0.1^{*}$ & 0.98 & $<0.001$ & 0.001 \\
\hline Obesity, \% & $39.5 \pm 0.7$ & $41.4 \pm 3.1$ & $29.6 \pm 1.8^{*}$ & 0.82 & $<0.001$ & 0.01 \\
\hline Smoking, \% & & & & 0.27 & $<0.001$ & $<0.001$ \\
\hline Never & $58.6 \pm 0.7$ & $53.7 \pm 2.9$ & $26.1 \pm 1.7$ & & & \\
\hline Ex- & $20.6 \pm 0.6$ & $23.1 \pm 2.7$ & $40.3 \pm 1.8^{*}$ & & & \\
\hline Current & $20.8 \pm 0.7$ & $23.2 \pm 2.7$ & $33.6 \pm 1.7^{*}$ & & & \\
\hline \multicolumn{7}{|l|}{ Comorbidities, $\%$} \\
\hline Hypertension & $37.7 \pm 0.8$ & $49.4 \pm 3.4^{*}$ & $53.2 \pm 1.8^{*}$ & 0.05 & $<0.001$ & 0.24 \\
\hline Diabetes & $11.2 \pm 0.5$ & $13.2 \pm 1.9$ & $17.1 \pm 1.4^{*}$ & 0.50 & $<0.001$ & 0.06 \\
\hline Hypercholesterolemia & $18.0 \pm 0.6$ & $20.1 \pm 2.6$ & $16.2 \pm 1.5$ & 0.41 & 0.26 & 0.17 \\
\hline Osteoarthritis & $16.2 \pm 0.6$ & $31.9 \pm 2.8^{*}$ & $17.8 \pm 1.5$ & $<0.001$ & 0.48 & $<0.001$ \\
\hline OA medication & $5.6 \pm 0.3$ & $13.5 \pm 2.2^{*}$ & $5.8 \pm 0.8$ & $<0.001$ & 0.69 & $<0.001$ \\
\hline \multicolumn{7}{|l|}{ Pulmonary function } \\
\hline FVC, L & $3.53 \pm 0.01$ & $3.22 \pm 0.06^{*}$ & $3.66 \pm 0.04^{*}$ & $<0.001$ & $<0.002$ & $<0.001$ \\
\hline FVC, \% & $93.4 \pm 0.2$ & $88.8 \pm 0.8^{*}$ & $91.5 \pm 0.5^{*}$ & $<0.001$ & $<0.001$ & 0.004 \\
\hline $\mathrm{FEV}_{1}, \mathrm{~L}$ & $2.81 \pm 0.01$ & $2.27 \pm 0.05^{*}$ & $2.34 \pm 0.03^{*}$ & $<0.001$ & $<0.001$ & 0.24 \\
\hline $\mathrm{FEV}_{1}, \%$ & $94.6 \pm 0.2$ & $82.7 \pm 1.1^{*}$ & $79.8 \pm 0.5^{*}$ & $<0.001$ & $<0.001$ & 0.02 \\
\hline $\mathrm{FEV}_{1} / \mathrm{FVC}, \%$ & $79.8 \pm 0.1$ & $70.3 \pm 0.7^{*}$ & $63.9 \pm 0.2^{*}$ & $<0.001$ & $<0.001$ & $<0.001$ \\
\hline \multicolumn{7}{|l|}{ EuroQOL } \\
\hline EQ-5D index & $0.94 \pm 0.002$ & $0.88 \pm 0.01^{*}$ & $0.92 \pm 0.01^{*}$ & $<0.001$ & $<0.001$ & 0.003 \\
\hline EQ-5D VAS & $75.6 \pm 0.5$ & $70.2 \pm 2.5^{*}$ & $73.4 \pm 1.4^{*}$ & 0.04 & 0.02 & 0.44 \\
\hline
\end{tabular}

* indicates clinical significance $(P<0.05)$

** $P$ values were analyzed with control groups (Asthma \& COPD group)

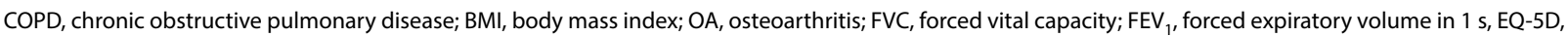
EuroQOL Five Dimensions Questionnaire; VAS, visual analogue scale

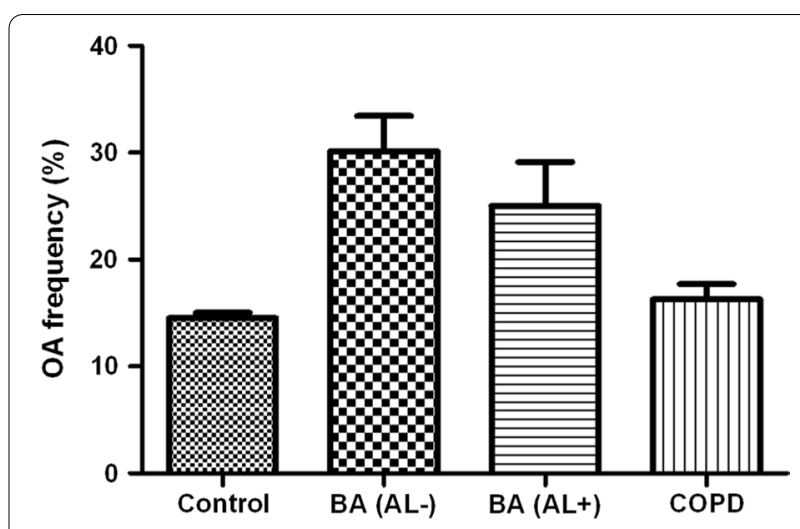

Fig. 2 Prevalence of osteoarthritis. BA, bronchial asthma; AL, airflow limitation; $C O P D$, chronic obstructive pulmonary disease; $O A$, osteoarthritis. Control vs. BA w/o AL, $P<0.001$; Control vs. BA w AL, $P=0.021$; Control vs. COPD, $P=0.48 ; \mathrm{BA} w / \mathrm{OAL}$ vs. BA $w \mathrm{AL}, P=0.14$; BA w/o AL vs. COPD, $P<0.001$; BA w AL vs. COPD, $P=0.091$ any effect of sex on this association, an interaction term was added to the previous multivariable model, but no significant interaction between sex and OA was noted $(P=0.43)$. Furthermore, to clarify the effect of obesity on this association, an interaction term between obesity and the presence of OA was added to model 1 and its interaction in the association with asthma without airflow limitation was not significant $(P=0.20)$. For sensitivity analysis, we reanalyzed the data, excluding patients with obesity, and the association between asthma and OA remained significant (OR, 1.70; 95\% CI, 1.07-2.70).

To examine the influence of $\mathrm{OA}$ medication on asthma, the multivariable analysis also included the current medical treatment for OA with adjusted variables (model 2). The presence of OA remained significantly associated with asthma (OR, 1.56; 95\% CI, 1.10-2.20), independent of the use of OA medication. Moreover, OA medication had no significant influence on the development of asthma $(P=0.63$; 
Table 2 Association of asthma and COPD with osteoarthritis

\begin{tabular}{llll}
\hline & OR & $\begin{array}{l}\text { Osteoarthritis } \\
\mathbf{9 5 \%} \mathbf{C l}\end{array}$ & $\boldsymbol{P}$ \\
\hline Asthma & 1.56 & $1.10-2.20$ & 0.013 \\
Without Airflow limitation & 1.97 & $1.32-2.93$ & $<0.001$ \\
With Airflow limitation & 0.94 & $0.46-1.89$ & 0.855 \\
COPD & 0.83 & $0.61-1.14$ & 0.220 \\
\hline
\end{tabular}

* Adjusted for age, sex, body mass index, and current smoking status COPD chronic obstructive pulmonary disease

Table 3 Comparison of characteristics of affected joints

\begin{tabular}{|c|c|c|c|}
\hline & $\begin{array}{l}\text { Controls } \\
(\mathrm{N}=7787)\end{array}$ & $\begin{array}{l}\text { Asthma } \\
(\mathrm{N}=425)\end{array}$ & $\begin{array}{l}\text { COPD } \\
(N=1131)\end{array}$ \\
\hline Knee pain, \% & $13.0 \pm 0.5$ & $26.0 \pm 2.8^{*}$ & $14.6 \pm 1.3$ \\
\hline Hip pain, \% & $6.1 \pm 0.3$ & $10.5 \pm 1.8^{*}$ & $6.5 \pm 0.9$ \\
\hline Back pain, \% & $14.8 \pm 0.6$ & $25.3 \pm 2.6^{*}$ & $18.2 \pm 1.5$ \\
\hline Radiographic OAf & $12.1 \pm 0.6$ & $20.5 \pm 2.9^{*}$ & $9.2 \pm 1.1^{*}$ \\
\hline \multicolumn{4}{|c|}{ Knee scale (KL grade)‡ } \\
\hline 0 & $44.2 \pm 1.1$ & $36.2 \pm 3.2$ & $40.6 \pm 2.0$ \\
\hline 1 & $22.9 \pm 0.8$ & $20.4 \pm 2.8$ & $27.3 \pm 1.9^{*}$ \\
\hline 2 & $13.0 \pm 0.6$ & $17.2 \pm 2.7^{*}$ & $15.4 \pm 1.4^{*}$ \\
\hline 3 & $13.5 \pm 0.7$ & $17.2 \pm 2.4^{*}$ & $11.4 \pm 1.3$ \\
\hline 4 & $6.4 \pm 0.5$ & $9.0 \pm 1.9^{*}$ & $5.3 \pm 1.0$ \\
\hline
\end{tabular}

* Indicates clinical significance $(P<0.05)$

FRadiographic evaluation was performed for the control ( $N=5261)$, asthma $(\mathrm{N}=353)$, and COPD $(\mathrm{N}=1051)$ groups in subjects $\geq 50$ years

COPD, chronic obstructive pulmonary disease; OA, osteoarthritis; KL grade, Kellgren-Lawrence grade

Table 2). Patients with asthma were stratified according to the presence of airflow limitation in model 2this association was only significant in patients with asthma without airflow limitation (Table 2). Since the development of airflow limitation could be affected by tobacco smoking, a sensitivity analysis that excluded current smokers was repeated and the presence of OA remained significantly associated with asthma (OR, 1.60; 95\% CI, 1.11-2.31), independent of the use of OA medication $(P=0.64)$.

To determine the difference among the various sites of $\mathrm{OA}$ in relation to asthma, a subanalysis for each location of pain was performed. The prevalence of knee, back, and hip pain were higher in patients with asthma than that in the control group, but not in patients with COPD (Table 3). The results of the univariable analysis are summarized in Table 4 . In the multivariable analysis, both knee and back pain were related to the presence of asthma, but hip pain was not significant $(P=0.30$; Table 4). Among the 9344 individuals, joint radiography was performed for 6674 , and the prevalence of radiographic knee OA using the KL grading system was $20.5 \% \pm 2.9 \%$ in the asthma group, $9.2 \% \pm 1.1 \%$ in the COPD group, and $12.1 \% \pm 0.6 \%$ in the control group. Severe knee OA (KL grade 3 or 4) was also more prevalent in the asthma group than in the COPD or control groups (Table 3 ). The radiographic KL score for the knee joint was significantly associated with the presence of asthma in both the univariable and multivariable analyses (Table 4). The radiographic KL score for the lumbar spine was related to the presence of asthma in the univariable analysis, but lost significance in the multivariable analysis $(P=0.53)$. The radiographic KL score for the hip joint was not significant in either the univariable $(P=0.26)$ or multivariable analyses $(P=0.65)$. The associations between asthma and the radiographic KL scores for each joint are summarized in Table 4.

Table 4 Association of the location of pain or radiographic severities of osteoarthritis with asthma and COPD

\begin{tabular}{|c|c|c|c|c|c|c|c|c|}
\hline & \multicolumn{4}{|c|}{ Asthma } & \multicolumn{4}{|c|}{ COPD } \\
\hline & \multicolumn{2}{|c|}{ Univariable } & \multicolumn{2}{|c|}{ Multivariable } & \multicolumn{2}{|c|}{ Univariable } & \multicolumn{2}{|c|}{ Multivariable } \\
\hline & OR & $95 \% \mathrm{Cl}$ & OR & $95 \% \mathrm{Cl}$ & OR & $95 \% \mathrm{Cl}$ & OR & $95 \% \mathrm{Cl}$ \\
\hline \multicolumn{9}{|l|}{ Pain site } \\
\hline Knee & 2.25 & $1.80-2.80$ & 1.78 & $1.40-2.26$ & 0.92 & $0.77-1.10$ & 0.78 & $0.64-0.96$ \\
\hline Back & 1.76 & $1.41-2.20$ & 1.37 & $1.08-1.74$ & 1.09 & $0.93-1.29$ & 0.96 & $0.79-1.15$ \\
\hline Hip & 1.54 & $1.11-2.12$ & 1.19 & $0.86-1.66$ & 0.94 & $0.74-1.21$ & 0.88 & $0.67-2.26$ \\
\hline \multicolumn{9}{|c|}{ Radiographic KL grade } \\
\hline Knee joint & 1.19 & $1.10-1.29$ & 1.10 & $1.01-1.21$ & 0.97 & $0.92-1.03$ & 0.94 & $0.88-1.00$ \\
\hline Lumbar spine & 1.25 & $1.08-1.46$ & 1.05 & $0.89-1.24$ & 1.49 & $1.36-1.64$ & 1.15 & $0.99-1.25$ \\
\hline Pelvis joint & 1.16 & $0.90-1.49$ & 1.06 & $0.82-1.38$ & 1.51 & $1.30-1.75$ & 0.95 & $0.81-1.12$ \\
\hline
\end{tabular}

COPD chronic obstructive pulmonary disease, KL Kellgren-Lawrence 


\section{Discussion}

The nationwide survey data collected from the general population revealed a significant correlation between asthma and OA. Despite the high prevalence of each of these conditions, there have been no reports describing their prevalence as comorbid conditions. Our study revealed the prevalence of $\mathrm{OA}$ in patients with asthma was as high as $31.9 \%$. Moreover, the prevalence of OA was higher in patients with asthma compared to that in healthy controls or in patients with COPD, which is a comparative respiratory disease. The relationship between OA and asthma was remarkable in the group without airflow limitations, and the radiographic severity of the knee joint also correlated with asthma. The lower QOL in the asthma group may be a consequence of its comorbidity with OA in this population.

OA and asthma mutually affect each other through several mechanisms. First, asthma has an immunological and inflammatory pathogenesis which could simultaneously affect the development of OA. There is strong evidence that endogenous and exogenous reactive oxygen and nitrogen species play major roles in airway inflammation, which determines the severity of asthma [19]. The oxidative stress described above is also known to be an important factor in the development of OA [20, 21]. Genome-wide association studies (GWAS) have shown that several single-nucleotide polymorphisms of the gene encoding SMAD family member 3 (SMAD3) have been reported to be associated with knee or hip OA in both the Caucasian and Asian populations [22-24]. SMAD3 is located on chromosome 15q21-22 and is known to have important anabolic effects on chondrocytes through intracellular messengers in the transforming growth factor- $\beta$ signaling pathway $[25,26]$. Remarkably, an epigenome-wide association study (EWAS) on asthma also reported differential methylation of inflammatory response-related genes, including SMAD3 [27]. Second, medication for OA, such as NSAIDs, could influence the development of asthma, although the independent effect of such medication on asthma development was not significant in the present study. Our study suggests that the association between asthma and NSAIDs could be attributable to the effect of OA instead of NSAIDs themselves, but further larger studies are needed to confirm our findings. Third, asthma and OA have common features that are influenced by age, sex, obesity, and tobacco smoking-these might act as confounders, although we tried to adjust for these effects in multiple ways in the multivariable analysis.

This study revealed that the knee joint in patients with OA showed the most significant relationship with asthma. Since $\mathrm{OA}$ is a degenerative joint disease, its mechanism is primarily related to weight bearing and/ or repetitive mechanical force; thus, the most commonly affected joints are known to be the hands and knees. However, the etiology of OA also includes the interaction between local tissue damage and the immune system, which may lead to chronic low-grade joint inflammation [28]. Altered levels of inflammatory mediators have been detected in OA synovial fluid suggesting synovial inflammation after meniscal damage [29]. Synovial joints are composed of diverse tissues that involve different loads, have distinct functional requirements, and possess differing proportional tissue types. The interaction of these factors may explain the preference of OA for certain anatomical sites, specifically the knees, hips, spine, hands, or feet [30]. Some reports have described strong genetic penetrance with diverse hereditary contribution, which is estimated to range from 30 to $65 \%$ depending on the joint sites [31-34]. Based on these studies, the association between asthma and OA, especially knee OA, could be explained, although we still cannot conclude whether there is a clear mechanism involved in this association.

The relationship between OA and asthma was found to be significant after adjusting for several confounding variables, and their association was more evident in the asthma group without airflow limitation. Asthma and COPD share similar phenotypic features and happen to be frequently misdiagnosed in clinical practice. Since patients with COPD may coexist in a group of patients with asthma with airflow limitation, the asthma group was split according to the presence of airflow limitation, and they presented distinct aspects of association with OA. In order to remove the patients with COPD that were part of the asthma group, we performed a sensitivity analysis excluding smokers and obtained a higher effect estimate.

Although the present study described the novel relationship between asthma and $\mathrm{OA}$, the following limitations of the study should be considered to facilitate the interpretation of our results. First, the prevalence of asthma was calculated using results from a self-reported questionnaire, not by a provocation test, which created the risk of misclassifying patients. Moreover, owing to the absence of a provocation test, we could not compare the severity correlation of these diseases, such as the value of provocative concentration causing a $20 \%$ drop in $\mathrm{FEV}_{1}$. Although our study included patients diagnosed by doctors, misdiagnoses or underdiagnoses could have still occurred, especially in patients with asthma accompanying airflow limitation, with COPD. Therefore, we tried to stratify patients with asthma according to the presence of airflow limitation, and the association with OA was evident in a relatively pure asthma group without airflow limitation. Second, we had a less than optimum amount 
of information about the involvement of OA in each patient, including its severity, radiological and clinical information, and treatment information. Moreover, it is possible that the patients with severe OA and a poor health condition were not included in this study because they could not attend a nationwide survey. Additionally, among the participants aged $\geq 40$ years, approximately $30 \%$ were excluded from the present analysis because they did not complete spirometry tests. Characteristic differences between these groups may affect selection bias. Finally, although OA medication alone was not associated with asthma in the subgroup analysis, we could not determine the exact type of medication used by the patients, such as steroids or NSAIDs. Further analyses are needed that focus on the differences based on the influence of the joint location and pattern of OA.

\section{Conclusions}

The OA in patients with asthma has a high prevalence. OA and asthma can be considered as risk factors for each other. Considering their comorbid characteristics, these patients need special attention in terms of physical activity and QOL in clinical practice. Further larger studies, based on GWAS and EWAS, or intervention studies are needed to confirm our findings.

\section{Supplementary Information}

The online version contains supplementary material available at https://doi. org/10.1186/s12890-021-01425-6.

Additional file 1. Composition of study population.

\section{Abbreviations}

BMI: Body mass index; COPD: Chronic obstructive pulmonary disease; EQ-5D: EuroQOL Five Dimensions Questionnaire; EWAS: Epigenome-wide association study; FEV: Forced expiratory volume in $1 \mathrm{~s}$; FVC: Forced vital capacity; GOLD: Global Initiative for Chronic Obstructive Lung Disease; GWAS: Genome-wide association study; KCDC: Korean Centers for Disease Control and Prevention; KL grade: Kellgren-Lawrence grade; KNHANES: Korean National Health and Nutrition Examination Survey; NSAIDs: Non-steroidal anti-inflammatory drugs; OA: Osteoarthritis; QOL: Quality of life.

\section{Acknowledgements}

Not applicable.

\section{Authors' contributions}

HKK, PS, and JHL preformed the literature review, conducted data analysis, and drafted the manuscript. They contributed to the interpretation of the data, critically revised the paper, and approved the final version. They developed the study conception, directed the analytic strategy of the study, and supervised the drafting of the manuscript. All authors approved the final manuscript as submitted and agree to be accountable for all aspects of the work. All authors read and approved the final manuscript.

\section{Funding}

This work was supported by the National Research Foundation of Korea (NRF) grant funded by the Korea government (MSIT; No. 2018RICIB5086591). The funding bodies played no role in the design of the study and collection, analysis, and interpretation of data and in writing the manuscript.

\section{Availability of data and materials}

The datasets generated and/or analyzed during the current study are publicly available through the Korean National Health and Nutrition Examination Survey webpage at https://knhanes.cdc.go.kr/knhanes/sub03/sub03_02_05.do.

\section{Ethics approval and consent to participate}

This study was conducted in accordance with the Declaration of Helsinki. The KNHANES protocols were reviewed and approved by the Research Ethics Review Board of National Center fot Health Statistics. Written informed consents were obtained from all KNHANES participants. Data was provided in public without personal indentification information.

\section{Consent for publication}

Not applicable.

\section{Competing interests}

The authors declare that they have no competing interests.

\section{Author details}

${ }^{1}$ Division of Pulmonary and Critical Care Medicine, Department of Internal Medicine, Inje University Ilsan Paik Hospital, Goyang, Korea. ${ }^{2}$ Department of Neurology, Inje University Ilsan Paik Hospital, Goyang, Korea. ${ }^{3}$ Division of Rheumatology, Department of Internal Medicine, Inje University Ilsan Paik Hospital, Goyang 10380, Korea.

Received: 20 September 2020 Accepted: 5 February 2021 Published online: 16 February 2021

\section{References}

1. Masoli M, Fabian D, Holt S, Beasley R. The global burden of asthma: executive summary of the GINA Dissemination Committee report. Allergy. 2004;59(5):469-78.

2. To T, Stanojevic S, Moores G, Gershon AS, Bateman ED, Cruz AA, Boulet LP. Global asthma prevalence in adults: findings from the cross-sectional world health survey. BMC Public Health. 2012;12:204

3. Reddel HK, Bateman ED, Becker A, Boulet LP, Cruz AA, Drazen JM, Haahtela T, Hurd SS, Inoue H, de Jongste JC, et al. A summary of the new GINA strategy: a roadmap to asthma control. Eur Respir J. 2015:46(3):622-39.

4. Amoah AS, Forson AG, Boakye DA. A review of epidemiological studies of asthma in Ghana. Ghana Med J. 2012;46(2 Suppl):23-8.

5. Sweeney J, Patterson CC, Menzies-Gow A, Niven RM, Mansur AH, Bucknall C, Chaudhuri R, Price D, Brightling CE, Heaney LG. Comorbidity in severe asthma requiring systemic corticosteroid therapy: cross-sectional data from the Optimum Patient Care Research Database and the British Thoracic Difficult Asthma Registry. Thorax. 2016;71(4):339-46.

6. Lawrence RC, Felson DT, Helmick CG, Arnold LM, Choi H, Deyo RA, Gabriel S, Hirsch R, Hochberg MC, Hunder GG, et al. Estimates of the prevalence of arthritis and other rheumatic conditions in the United States. Part II Arthritis Rheum. 2008:58(1):26-35.

7. Moskowitz RW. The burden of osteoarthritis: clinical and quality-of-life issues. Am J Manag Care. 2009;15(8 Suppl):S223-9.

8. Blanca-Lopez N, Soriano V, Garcia-Martin E, Canto G, Blanca M. NSAIDinduced reactions: classification, prevalence, impact, and management strategies. J Asthma Allergy. 2019;12:217-33.

9. Vestbo J, Hurd SS, Rodriguez-Roisin R. The 2011 revision of the global strategy for the diagnosis, management and prevention of COPD (GOLD)—why and what? Clin Respir J. 2012;6(4):208-14.

10. Mancia G, Fagard R, Narkiewicz K, Redon J, Zanchetti A, Bohm M, Christiaens T, Cifkova R, De Backer G, Dominiczak A, et al. 2013 ESH/ESC Guidelines for the management of arterial hypertension: the Task Force for the management of arterial hypertension of the European Society of Hypertension (ESH) and of the European Society of Cardiology (ESC). J Hypertens. 2013;31(7):1281-357.

11. Standards of medical care in diabetes_-2013. Diabetes Care. 2013;36 Suppl 1:S11-66. 
12. Third report of the National Cholesterol Education Program (NCEP) expert panel on detection, evaluation, and treatment of high blood cholesterol in adults (Adult Treatment Panel III) final report. Circulation. 2002;106(25):3143-421.

13. WHO Expert Consultation. Appropriate body-mass index for Asian populations and its implications for policy and intervention strategies. Lancet. 2004;363(9403):157-63.

14. Standardization of Spirometry, 1994 Update. American Thoracic Society. Am J Respir Crit Care Med. 1995:152(3):1107-36.

15. Choi JK, Park D, Lee JO. Normal predictive values of spirometry in Korean population. Tuberc Respir Dis. 2005;58:230-42.

16. Jordan JM, Helmick CG, Renner JB, Luta G, Dragomir AD, Woodard J, Fang F, Schwartz TA, Abbate LM, Callahan LF, et al. Prevalence of knee symptoms and radiographic and symptomatic knee osteoarthritis in African Americans and Caucasians: the Johnston County Osteoarthritis Project. J Rheumatol. 2007;34(1):172-80.

17. Kellgren JH, Lawrence JS. Radiological assessment of osteo-arthrosis. Ann Rheum Dis. 1957;16(4):494-502.

18. EuroQol-a new facility for the measurement of health-related quality of life. Health Policy. 1990;16(3):199-208.

19. Rambacher KM, Moniri NH. The $\beta 2$-adrenergic receptor-ROS signaling axis: an overlooked component of $\beta 2 A R$ function? Biochem Pharmacol. 2020;171:113690.

20. Li D, Xie G, Wang W. Reactive oxygen species: the 2-edged sword of osteoarthritis. Am J Med Sci. 2012;344(6):486-540.

21. Henrotin Y, Kurz B, Aigner T. Oxygen and reactive oxygen species in cartilage degradation: friends or foes? Osteoarthritis Cartilage. 2005;13(8):643-54.

22. Yang HY, Hu WH, Jiang T, Zhao H. SMAD3 gene rs 12901499 polymorphism increased the risk of osteoarthritis. Biosci Rep. 2018;38(3):BSR20180380.

23. Zhang $L$, Zhang $H$, Wang W, Zhao Y. Association between SMAD3 gene rs12901499 polymorphism and knee osteoarthritis in a Chinese population. J Clin Lab Anal. 2018;32(5):e22383.

24. Zhong F, Lu J, Wang Y, Song H. Genetic variation of SMAD3 is associated with hip osteoarthritis in a Chinese Han population. J Int Med Res. 2018;46(3):1178-86.
25. Li Y, Schang G, Boehm U, Deng CX, Graff J, Bernard DJ. SMAD3 regulates follicle-stimulating hormone synthesis by pituitary gonadotrope cells in vivo. J Biol Chem. 2017;292(6):2301-14.

26. Furumatsu T, Tsuda M, Taniguchi N, Tajima Y, Asahara H. Smad3 induces chondrogenesis through the activation of SOX9 via CREB-binding protein/p300 recruitment. J Biol Chem. 2005;280(9):8343-50.

27. Edris A, den Dekker HT, Melen E, Lahousse L. Epigenome-wide association studies in asthma: a systematic review. Clin Exp Allergy. 2019;49(7):953-68

28. Sokolove J, Lepus CM. Role of inflammation in the pathogenesis of osteoarthritis: latest findings and interpretations. Ther Adv Musculoskelet Dis. 2013;5(2):77-94.

29. Loeser RF, Goldring SR, Scanzello CR, Goldring MB. Osteoarthritis: a disease of the joint as an organ. Arthritis Rheum. 2012;64(6):1697-707.

30. Mimpen JY, Snelling SJB. Chondroprotective Factors in Osteoarthritis: a Joint Affair. Curr Rheumatol Rep. 2019;21(8):41.

31. Thomas E, Peat G, Croft P. Defining and mapping the person with osteoarthritis for population studies and public health. Rheumatology (Oxford). 2014;53(2):338-45.

32. Jordan JM, Kraus VB, Hochberg MC. Genetics of osteoarthritis. Curr Rheumatol Rep. 2004;6(1):7-13.

33. Kraus VB, Jordan JM, Doherty M, Wilson AG, Moskowitz R, Hochberg M, Loeser R, Hooper M, Renner JB, Crane MM, et al. The Genetics of Generalized Osteoarthritis (GOGO) study: study design and evaluation of osteoarthritis phenotypes. Osteoarthritis Cartilage. 2007;15(2):120-7.

34. Spector TD, Cicuttini F, Baker J, Loughlin J, Hart D. Genetic influences on osteoarthritis in women: a twin study. BMJ. 1996;312(7036):940-3.

\section{Publisher's Note}

Springer Nature remains neutral with regard to jurisdictional claims in published maps and institutional affiliations.
Ready to submit your research? Choose BMC and benefit from:

- fast, convenient online submission

- thorough peer review by experienced researchers in your field

- rapid publication on acceptance

- support for research data, including large and complex data types

- gold Open Access which fosters wider collaboration and increased citations

- maximum visibility for your research: over $100 \mathrm{M}$ website views per year

At $\mathrm{BMC}$, research is always in progress.

Learn more biomedcentral.com/submissions 